\title{
Can We Constrain Solar Interior Physics by Studying the Gravity-Mode Asymptotic Signature?
}

\author{
R.A. García $\cdot$ S. Mathur $\cdot$ J. Ballot
}

Received: 18 October 2007 / Accepted: 29 February 2008 / Published online: 28 March 2008

(C) Springer Science+Business Media B.V. 2008

\begin{abstract}
Gravity modes are the best probes to infer the properties of the solar radiative zone, which represents $98 \%$ of the Sun's total mass. It is usually assumed that highfrequency $g$ modes give information about the structure of the solar interior whereas lowfrequency $g$ modes are more sensitive to the solar dynamics (the internal rotation). In this work, we develop a new methodology, based on the analysis of the almost constant separation of the dipole gravity modes, to introduce new constraints on the solar models. To validate this analysis procedure, several solar models - including different physical processes and either old or new chemical abundances (from, respectively, Grevesse and Noels (Origin and Evolution of the Elements 199, Cambridge University Press, Cambridge, 15, 1993) and Asplund, Grevesse, and Sauval (Cosmic Abundances as Records of Stellar Evolution and Nucleosynthesis CS-336, Astron. Soc. Pac., San Francisco, 25 - 38, 2005)) - have been compared to another model used as a reference. The analysis clearly shows that this methodology has enough sensitivity to distinguish among some of the models, in particular, among those with different compositions. The comparison of the models with the $g$-mode asymptotic signature detected in GOLF data favors the ones with old abundances. Therefore, the physics of the core - obtained through the analysis of the $g$-mode properties - is in agreement with the results obtained in the previous studies based on the acoustic modes, which are mostly sensitive to more external layers of the Sun.
\end{abstract}

Keywords Helioseismology: Observations · Interior: Radiative zone, core

Helioseismology, Asteroseismology, and MHD Connections

Guest Editors: Laurent Gizon and Paul Cally.

R.A. García $(\bowtie) \cdot$ S. Mathur

Laboratoire AIM, CEA/DSM-CNRS, U. Paris Diderot, IRFU/SAp, 91191 Gif-sur-Yvette Cedex, France e-mail: rgarcia@cea.fr

S. Mathur

e-mail: smathur@cea.fr

J. Ballot

Max-Planck-Institut für Astrophysik, Karl-Schwarzschild-Strasse 1, 85748 Garching, Germany

e-mail: jballot@mpa-Garching.mpg.de 


\section{Introduction}

The combination of detailed modeling and helioseismic observations has enabled us to accurately describe the solar interior from the surface to its center where the nuclear reactions take place. The precise characterization of the resonant acoustic (pressure or $p$ ) modes has allowed us to constrain the theories describing the solar interior. The profiles of sound speed, density (e.g., Basu et al., 1997; Couvidat, Turck-Chièze, and Kosovichev, 2003), and differential rotation (Thompson et al., 1996; Howe et al., 2000; Chaplin et al., 2001; Couvidat et al., 2003), as well as the position of the base of the convective zone (e.g., Christensen-Dalsgaard, Gough, and Thompson, 1991) and the prediction of the neutrino fluxes (e.g., Turck-Chièze et al., 2001) are some examples of the constraints provided by the $p$-mode analyses.

Unfortunately, only a few $p$ modes propagate inside the inner core - below 0.25 solar radius $\left(R_{\odot}\right)$ - which contains about $50 \%$ of the solar mass. To make progress in knowledge of these layers, another type of mode is needed: the gravity $(g)$ modes. These modes have not yet been unambiguously detected individually in the Sun (for example, see Appourchaux et al., 2000; Gabriel et al., 2002; Elsworth et al., 2006) because they become evanescent in the convection zone, reaching the surface of the Sun with very small amplitudes (Andersen, 1996; Kumar, Quataert, and Bahcall, 1996). Some candidates have been detected (Turck-Chièze et al., 2004b; Mathur et al., 2007) by analyzing helioseismic data from the Global Oscillations at Low Frequencies (GOLF; Gabriel et al., 1995) instrument onboard the ESA/NASA Solar and Heliospheric Observatory (SOHO; Domingo, Fleck, and Poland, 1995) spacecraft. These candidates have provided some scenarios about the possible dynamics of the solar core but it has been impossible to correctly label these peaks in terms of their orders and degrees $(n, \ell, m)$ and to give the central frequencies of the candidates. Recently, García et al. (2007) were able to uncover the signature of the asymptotic properties of the dipole $(\ell=1) \mathrm{g}$ modes by measuring their constant spacing $\Delta P_{1}$. Indeed, for a given degree $\ell$, the difference $\Delta P_{\ell}$ of the periods of $g$ modes with consecutive radial order $n$ is almost constant when $n \gg \ell$ (Tassoul, 1980; Provost and Berthomieu, 1986). The comparison of the observations with some solar models - including different rotation of the core - showed the great sensitivity of this analysis to the dynamics of the solar core and it implied a rotation rate in the core faster than in the rest of the radiative region.

During the past few decades, many new physical processes have been studied and included in the solar models, whereas others have been refined to better match the helioseismic observations. Today, these models include better descriptions of the microscopic physics such as opacities, equation of state, nuclear reaction rates (e.g., Morel, Provost, and Berthomieu, 1997 and references therein), microscopic diffusion (Michaud and Proffitt, 1993) as well as other processes, for example turbulence mixing in the tachocline (Brun, Turck-Chièze, and Zahn, 1999).

New studies of the solar spectrum using the most recent advances in 3D modeling of the solar atmosphere and taking into account nonlocal thermodynamic equilibrium (NLTE) effects have provided new estimations of the solar photospheric composition (Asplund, Grevesse, and Sauval, 2005). In comparison with the older estimations (e.g., Grevesse and Noels, 1993) based on 1D models and the LTE approximation, this new measurement leads to a smaller metallicity and to a substantial modification of the abundances of $\mathrm{C}, \mathrm{N}$, and $\mathrm{O}$, which are crucial in solar modeling. The standard models computed with these new abundances yield a larger discrepancy from the helioseismic observations. They disagree with sound-speed and density profiles inferred from acoustic modes (for example, see TurckChièze et al., 2004a; Bahcall, Serenelli, and Basu, 2005; Guzik, Watson, and Cox, 2005) 
and we need, for example, to modify some opacities to reduce the discrepancy. It has also been shown that the models computed with these new abundances modify the frequencies of the resonant low-degree $p$ and $g$ modes (Basu et al., 2007; Chaplin et al., 2007; Zaatri et al., 2007; Mathur et al., 2007).

In this paper we use a set of solar models (briefly described in Section 2) - including different physical processes combined with the old and new abundances of the Sun and with different core rotation rates - that we utilize as a tool to study whether the methodology used to detect the dipole gravity modes in the Sun by García et al. (2007) (and summarized in Section 3) can distinguish among these models. To do so, in Section 4 we compare all of them to our reference model: the Saclay seismic model (Turck-Chièze et al., 2001). Finally, once the method has been demonstrated, we compare, in Section 5, the solar models with the GOLF data.

\section{Description of the Solar Models}

Several solar models, which differ from each other by the physical processes that they include, have been computed with the Code d'Évolution Stellaire Adaptatif et Modulaire (CESAM) (Morel, 1997). They have been developed to study the influence of different physical processes and the impact of the new abundances on the $g$-mode frequencies. These models are fully explained in Mathur et al. (2007). Here we recall briefly their main characteristics.

First let us describe the solar model that has been taken as a reference: the so-called seismic model (corresponding to the model seismic of Couvidat, Turck-Chièze, and Kosovichev, 2003). It is important to note that this model is produced by an evolutionary code and is not directly deduced from a seismic inversion. It is "seismic" because some physical quantities, such as opacities or nuclear reaction rates, have been tuned to better reproduce the soundspeed profile in the radiative zone deduced from the analysis of $p$-mode observations. One of its aims was to improve the prediction of the neutrino flux. This model includes turbulent diffusion in the tachocline and the chemical composition of Grevesse and Noels (1993).

As we want to compare the impact of different physical processes in our work, five other solar models have been used. They mainly differ in three physical inputs: microscopic diffusion (including the gravitational settling), horizontal turbulent diffusion in the tachocline, and abundances of chemical elements. The model called no diffusion does not include any diffusion processes, whereas all of the following models include diffusion through the prescription of Michaud and Proffitt (1993). Although today this model is obsolete, it is a good test for the detection methodology, since the $g$-mode frequencies are very different as compared to the seismic ones and therefore the analysis should be able to clearly separate them.

The second process, tachocline mixing, is introduced in the code following the prescription of Spiegel and Zahn (1992). The models including this process are called tacho whereas the ones without this turbulent effect are named std. This turbulent horizontal motion contributes to the thinness of the tachocline responsible for the mixing of chemical elements. Finally, the chemical composition is either taken from Grevesse and Noels (1993) (GN93 hereafter) or from the recent release of Asplund, Grevesse, and Sauval (2005) (As05 hereafter). The difference between these two chemical abundances is a decrease of the heavy element by $\approx 30 \%$. Such differences substantially affect, for instance, the Rossland opacities and lead to noticeably different thermal stratifications. The models using the old abundances have the suffix 93 whereas the models including the new abundances have the suffix 05 . Table 1 summarizes the main characteristics of all of the models used. Finally, to use a model calculated with another stellar evolution code, the following study is also done with model S 
Table 1 The main characteristics of the solar evolution models used to compute the $g$-mode frequencies

\begin{tabular}{lllll}
\hline Solar model & $\begin{array}{l}\text { Microscopic } \\
\text { diffusion }\end{array}$ & $\begin{array}{l}\text { Diffusion in } \\
\text { tachocline }\end{array}$ & GN93 & As05 \\
\hline
\end{tabular}

\begin{tabular}{lllll} 
no diffusion & & & $\times$ & \\
std93 & $\times$ & & $\times$ & \\
std05 & $\times$ & $\times$ & & $\times$ \\
tacho93 & $\times$ & $\times$ & & \\
tacho05 & $\times$ & & & $\times$ \\
\hline
\end{tabular}

from Christensen-Dalsgaard et al. (1996), which is a standard model including microscopic diffusion and using GN93.

From each stellar structure, eigenfrequencies have been computed with the adiabatic pulsation package (adipack, provided by J. Christensen-Dalsgaard and available on http:// www.phys.au.dk/ jcd/adipack.n/). The computation of $g$-mode frequencies can become very sensitive to the numerical approach, especially to the treatment of the boundary condition at the center. Therefore, the numerical uncertainties on the computed $g$-mode periods become large at very low frequency and we should be extremely cautious. However, in the range used for this study (between 2 and 8.5 hours; see Section 3), it has been shown that, for a given model, frequencies are modified by less than $50 \mathrm{nHz}$ by changing the numerics (see Mathur, 2007), leading to a maximum uncertainty of around 50 seconds on the mode periods. Such errors are smaller than the observed deviations among the different models used here. In fact, they are too small (by more than an order of magnitude) to be correctly distinguished by the methodology developed in this paper.

It is usually considered that the high-frequency $g$ modes (above $150 \mu \mathrm{Hz}$ ) are very sensitive to the physical processes included in the models (the structure) giving up to $5 \mu \mathrm{Hz}$ of difference among the models, whereas at lower frequencies - inside the asymptotic region the frequency differences are smaller than a fraction of a microhertz and they could be more useful for studying the dynamics of the solar interior (for example, see Mathur et al., 2007). Indeed, we have already seen that the difference of the periods of two consecutive $g$ modes with the same degree $(\ell)$ and successive radial order $(n)$ is almost constant. In Figure 1 the differences $\left(\Delta P_{1}\right)$ of the central frequencies ( $m=0$ components) of the $g$ modes have been plotted for all the models described in Table 1 . The asymptotic regime is reached for periods above $\approx$ five hours and the differences among all of the models considered here are within one minute (from 24 to 25 minutes). Thus, it is going to be very difficult to distinguish among all these models.

Moreover, until now we have only considered the effect of the structure on the $g$-mode frequencies but we cannot neglect the effect of the rotation of the solar interior on the other $m$-components of the modes. Indeed, in Figure 1 we have also drawn the $m \pm 1$ components for one of the solar models, the std05, by considering a core rotating as a rigid body, like the rest of the radiative region. The dispersion in $\Delta P_{1}$ introduced by the dynamics (without even considering a rapid rotation in the core, just a rigid rotation profile) is already of the same order of the structural effects owing to the different physical processes and abundances considered. That means that the two effects cannot be easily disentangled and we have to study both at the same time.

At low frequency, it seems more appropriate to study the $g$ modes in period, and not in frequency. Depending on the physical processes taken into account in the solar models, there can be important differences among the periods of the $g$ modes. Thus, we could expect to 
Figure 1 Separations in period $\left(\Delta P_{1}\right)$ of the $m=0$ components of the $g$ modes computed by using the models described in Table 1. For clarity, we have plotted the $m \pm 1$ components only for one model: the $s t d 05$ (with a solid rotation of $\Omega_{r z}=433 \mathrm{nHz}$ in the radiative zone and a rotation in the convection zone that reproduces the inferred profile deduced from helioseismology data).

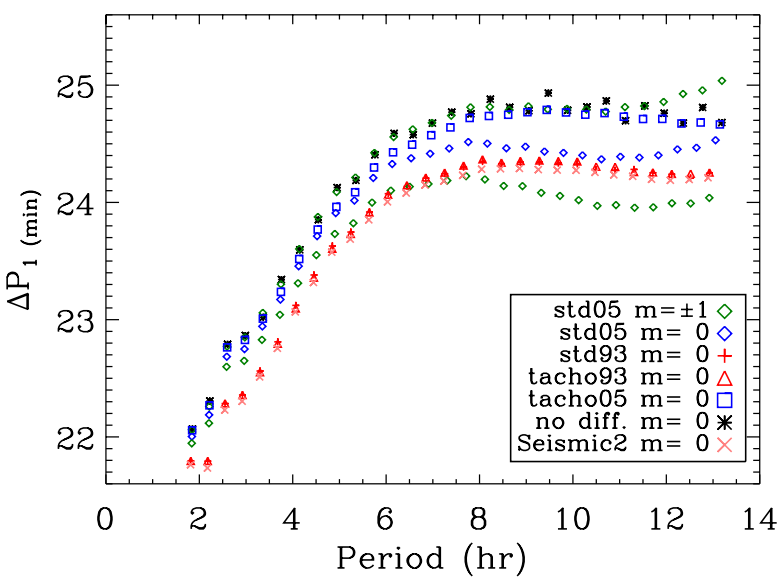

use these low-frequency $g$ modes to diagnose some physical processes or abundances taken into account in the models.

To complete this study we need to model the dynamics - the rotation rate - of the solar interior, in particular, in the regions where the $g$ modes are trapped (i.e. the radiative region). Today, the solar rotation rate is known well down to the solar core (Thompson et al., 2003) but it is still uncertain in the deepest layers. We have thus computed the $g$-mode splittings corresponding to ten artificial rotation profiles $\Omega(r, \theta)$. These artificial profiles have a differential rotation in the convection zone (that mimics the real behavior) and a rigid rotation from 0.7 down to a given fractional radius $r_{i}=r / R_{\odot}(0.1,0.15$, and 0.2$)$, which is equal to $\Omega_{\mathrm{rad}}=433 \mathrm{nHz}$. From each of these internal fractional radii $\left(r_{i}\right)$ down to the center we have a step-like profile having a rate of three, five, and ten times larger than the rest of the radiative zone. Finally, we have also computed a rigid rotation rate down to the center of the Sun, which is considered as our reference rotation profile.

\section{Methodology}

To compare the signature of the $g$-mode asymptotic properties between real and modeled spectra or even between two different synthetic spectra, we have used the method developed by García et al. (2007). Indeed, a numerical simulation of the $g$-mode power spectrum has been used (see Section S.2 of the on-line material of García et al., 2007, for a full description of the simulation). Briefly, the central frequencies of the $g$ modes have been computed as described in Section 2. Their amplitudes have been considered to all be the same following the calculations done by Kumar, Quataert, and Bahcall (1996), where it has been shown that all the modes in the studied regions have roughly the same amplitudes. We have also simulated the $g$-mode amplitudes following different laws (e.g., a linear variation or a parabolic decreasing with frequency). In all the cases, the results were qualitatively the same. Finally, one-bin line widths have been used but we have checked that the results do not significantly change when wider modes are simulated. Since we are only interested in the power spectrum, the mode-phase information is lost. It is also important to note that none of the computed simulations contain noise because otherwise we could not quantify those effects coming from the models and those coming from the different noise realizations. Therefore, this study represents what we could obtain in the best conditions. The addition 

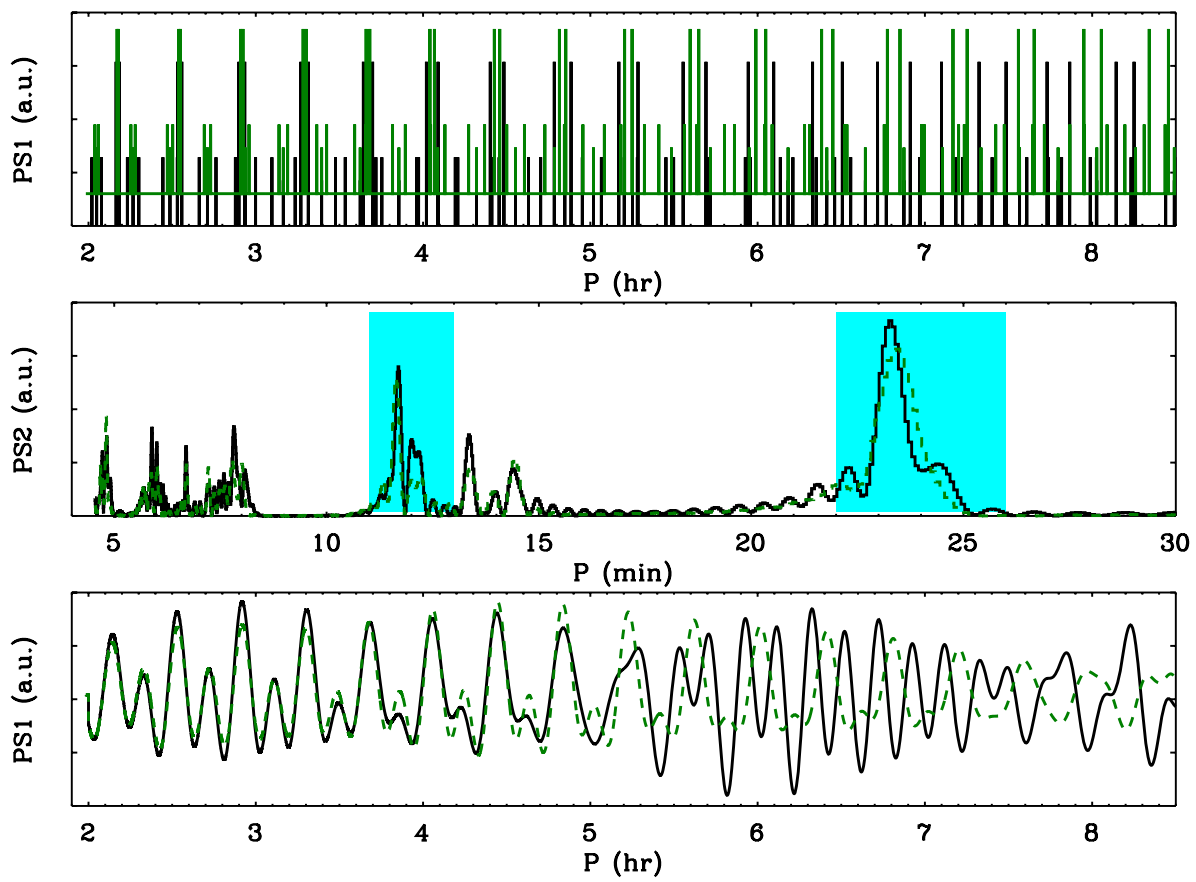

Figure 2 Top panel: Power spectral density, PS1, of $\ell=1$ and 2 modes computed by using the seismic model and two rotation laws in the core: a rigid core (shifted upward in green) and a core rotating five times faster than the rest of the radiative zone at $r_{i}=0.15$ (continuous black lines in all the panels). Middle panel: Power spectral density, PS2, computed from PS1 between 2 and 11 hours. The shaded regions are those used to reconstruct the signal in PS1. Bottom panel: Reconstructed signals in PS1. In the two lower panels, the green dashed lines correspond to the case of a rigid core rotation law.

of noise (both instrumental and solar) would affect the data and could probably degrade the results.

The starting point of this analysis is the oscillation power spectrum density. We will call it hereafter the first spectrum (PS1). The real first spectrum is computed from GOLF time series calibrated into velocity (García et al., 2005) with a classical periodogram; the synthetic ones are directly built from the $g$-mode frequencies and rotational splittings derived from the considered models (see top panel of Figure 2).

PS1 is expressed as a function of the period, since the $g$ modes present a comblike structure in period and not in frequency, as is the case for $p$ modes. We have then performed a spectral analysis of the first spectrum, limited to the range $2-11$ hours (i.e., $25-140 \mu \mathrm{Hz}$ ), to get the second spectrum (hereafter called PS2). It is important to note that the considered PS2 is a complex spectrum, which is required to keep information on both amplitude and phase. For practical purposes, as PS1 is not regularly sampled in period, we cannot use a classical fast Fourier transform (FFT) and we use instead a general method based on sine-wave fittings.

The asymptotic signature of the $\ell=1 \mathrm{~g}$ modes is detected in $|\mathrm{PS} 2|^{2}$ as a high and broad peak around 22 to 25 minutes. Depending on the physics of the solar model and on the rotation rate in the radiative region, the shape and the position of the $\Delta P_{1}$ pattern is modified. Thus in the middle panel of Figure 2 we show the resultant PS2 of the same seismic model but using two different rotation rates in the core, a rigid rotation in the whole radiative zone 
and a rotation rate five times faster than the rest of the radiative zone starting at $r_{i}=0.15$. The model with the higher rotation rate has more power at 24.5 minutes and widens the $\Delta P_{1}$ structure.

Once this signature (a pattern of peaks) has been detected in PS2, we can locate in PS1 what contributes to the emergence of the structure in the PS2. To do so, we select in PS2 the vicinity around the pattern at $\Delta P_{1}$ and around its first harmonic at $\frac{1}{2} \Delta P_{1}$. We reconstruct the sum of the sine waves corresponding to the amplitudes, frequencies, and phases measured in PS2 in these limited regions. In other words, we perform an inverse Fourier transform of PS2 after having applied a narrow-band filter around what we consider to be the $\ell=1 \mathrm{~g}$-mode signature and its first harmonic.

The maxima of these reconstructed waves indicate the location in PS1 corresponding to the positions where we found peaks that have a repetitive pattern. If this signature is indeed the one of the dipolar $g$ modes, the maxima of these reconstructed waves correspond to the $m$-components - depending on their splittings - of the $\ell=1 \mathrm{~g}$ modes. The bottom panel of Figure 2 shows the reconstructed signals of the seismic models and the same two rotation rates in the core as in the previous example. At low periods, less than five hours, the reconstructed waves are nearly the same in both cases, but at higher periods, the model with a higher rotation rate in the core follows the separation of the two $m$-components owing to the higher splittings and the position of the maxima are different compared to the model using the rigid rotation profile.

Finally, we need to quantify the consistency between our models and the GOLF observations. Comparing $\Delta P_{1}$ is not very informative because, as mentioned by Mathur et al. (2007), all of the theoretical computations of $\Delta P_{1}$ are in agreement to within a minute and, unfortunately, there is not enough resolution to reach such an accuracy in PS2. However, a direct comparison of the periods of the modes shows clear discrepancies between the different models (see bottom panel of Figure 2). Thus, to compare the positions of the modes in PS1, we compute the Pearson's correlation coefficients (e.g., see Press et al., 1992) between the reconstructed waves of the models and the reference one (Section 4) or with the GOLF data (Section 5). If the correlation coefficient is clearly positive, then the maxima of both reconstructed waves coincide generally; that is, in both considered first spectra (real and synthetic or both synthetic), the $g$-mode components are overall at the same position. If the modes in one spectrum are shifted compared to the modes in the other one, the correlation coefficient decreases to zero and even becomes negative. A large negative correlation indicates that each mode component in one spectrum is in between two mode components of the other spectrum.

The range used for the correlation analysis has been restricted to between 2 and 8.5 hours (33 to $140 \mu \mathrm{Hz}$ ). The reason for reducing to the low-frequency limit is twofold: On one hand, the dynamical effects on the periods are so important when high core rotation rates are taken into account that the $m$-components of different modes $\ell=1$ and consecutive orders $(n)$ are mixed and the interpretation of the results is more complicated; on the other hand, the calculation of the frequencies at such rapid periods (above nine hours) could be less reliable and numerical effects can play an important role, as has been stated in the previous section.

\section{Comparison with the Seismic Model}

We have seen in the previous sections that the period of the $g$ modes in the low-frequency region (below $150 \mu \mathrm{Hz}$ ) is dominated by the physics and the dynamics of the radiative region, and mainly inside the solar core. To study the influence of the dynamics on the correlation coefficients, we apply the methodology described in the previous section to the seismic 


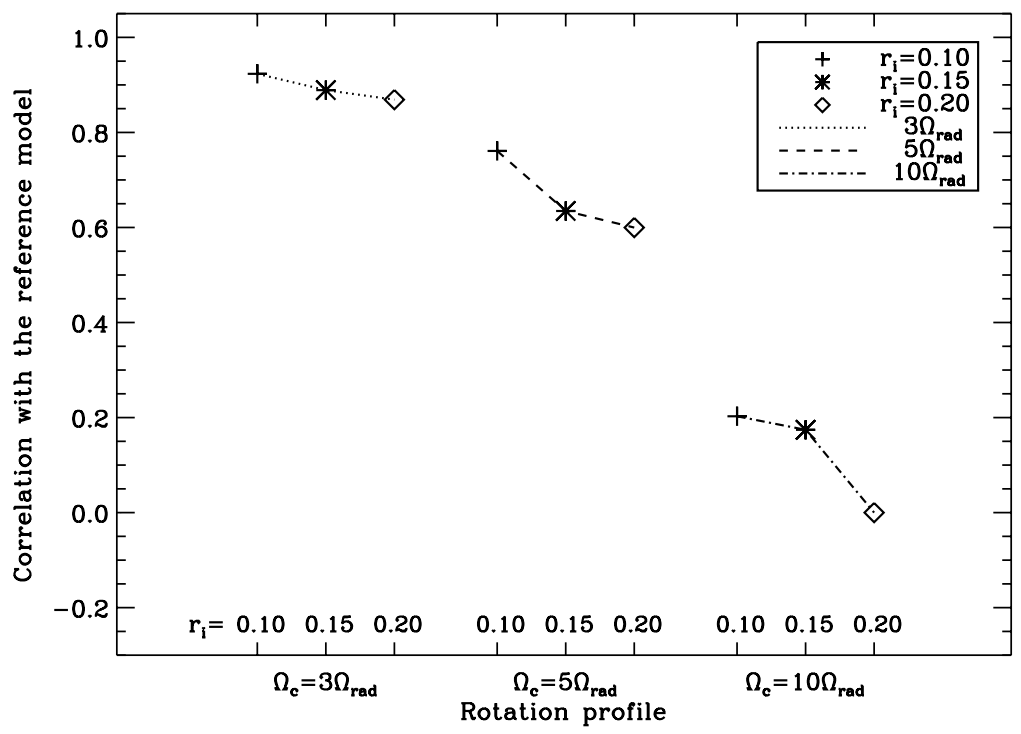

Figure 3 Correlation coefficients between the reconstructed waves for the seismic model with a rigid rotation profile and the ones for the seismic model with other rotation profiles. All of the rotation profiles have a differential rotation in the convective zone and a rigid rotation from 0.7 down to $r_{i}\left(=r / R_{\odot}\right)$ of 0.1 (crosses), 0.15 (asterisks), and 0.2 (diamonds). The rotation rate below $r_{i}$ is constant: $3 \Omega_{\mathrm{rad}}$ (dotted line), $5 \Omega_{\mathrm{rad}}$ (dashed line), and $10 \Omega_{\mathrm{rad}}$ (dotted-dashed line), where $\Omega_{\mathrm{rad}}$ is the rotation rate in the rest of the radiative zone $(433 \mathrm{nHz})$.

model with a rigid rotation profile that is taken as our reference model. Then, we compute the correlation coefficient between the waves reconstructed for this reference model and the ones for the seismic model with different rotation profiles. The results are shown in Figure 3.

We can note that the higher the rotation rate in the core, the lower the value of the correlation will be (i.e., when the difference between the reference and the studied model is bigger, the correlation is smaller). For a given rotation rate in the core, if we shift the value of $r_{i}$ from $0.1 R_{\odot}$ to $0.2 R_{\odot}$, the correlation decreases a little, showing that there is low sensitivity to the fractional radius $r_{i}$. Then if we had only one reliable model with defined physical processes, we could infer some information on the rotation profile from this correlation. We would be able to say, for instance, that a very rapid rotation in the core is very unlikely to be compatible with our reference model (seismic with rigid rotation profile).

However, another parameter has to be taken into account: the solar structure. In Figure 4, we have plotted the correlation coefficients between the reconstructed waves for the seismic model with a given rotation profile and another model with the same rotation profile. As each point on the plot is calculated with two different models but with the same rotation profile, it indicates the sensitivity of the correlation to the different physical parameters included in the two compared models.

We can see in Figure 4 that there are two clearly separated groups of correlations. The first one contains the models with the old abundances of GN93 that give correlations above $70 \%$ whatever rotation profile to which they are attributed. The second group of models, containing the model without diffusion and the models that include the new abundances of As05, gives correlations below 30\% and even negative. Thus the $g$-mode frequencies extracted from these two groups are incompatible. Although this result is already known (Zaatri et al., 2007; Mathur et al., 2007), it proves the ability of this methodology to separate 


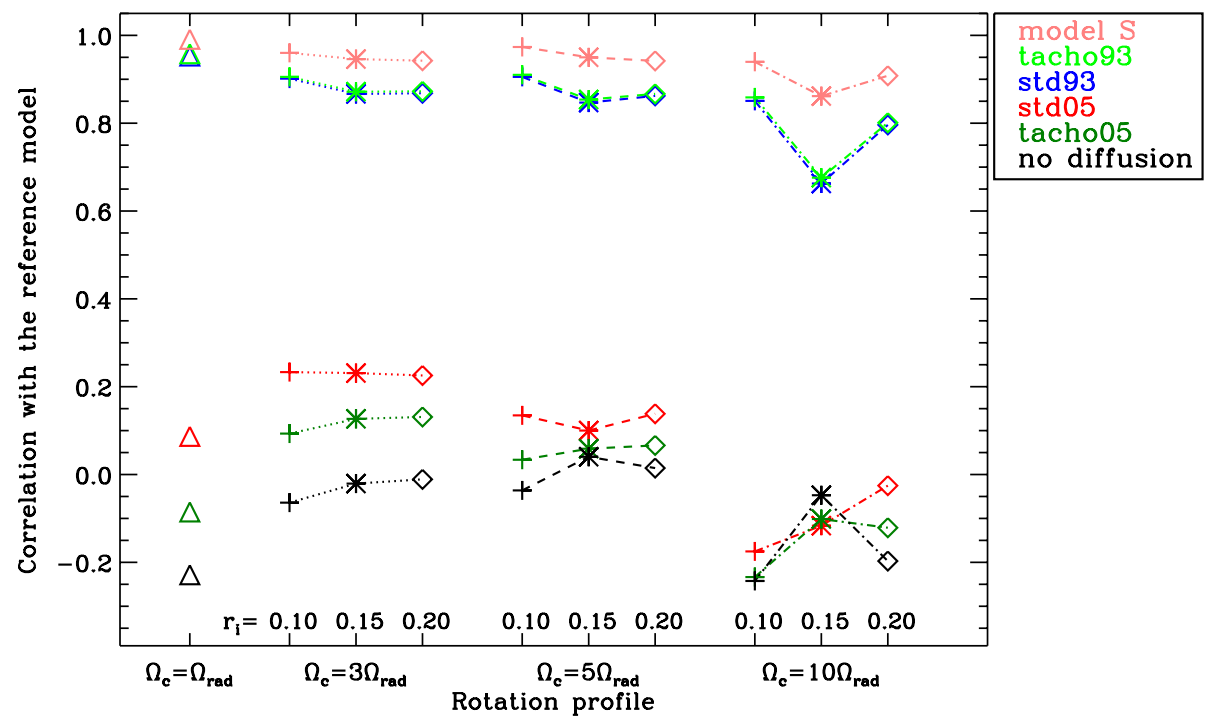

Figure 4 Correlation coefficients between the reference model (seismic) with a given rotation profile depending on the rate in the region below $r_{i}$ as described in Figure 3 (plus the rigid rotation profile (triangles)) and another model with the same rotation profile. These models (described in Section 2) are std93 (blue), std05 (red), tacho93 (light green), tacho05 (dark green), no diffusion (black), and model S (pink).

the two types of models. Obviously, there are tiny differences among other models such as the std93 and the tacho93 models because the frequency differences between the $g$ modes computed from them are very small.

Moreover, we can see other systematics in Figure 4 as the correlation coefficient decreases with higher rotation rates in the core. When higher rotation rates are considered, the reconstructed waves have to match both $m \pm 1$ components of the $\ell=1$ modes. Therefore, the structure of the waves is more complicated, allowing a higher precision in the calculation of the correlation and more sensitivity to the positions of the modes (the structure parameters of the model).

\section{Comparison with the Real Data}

To compare our models with the real data, we use the 3481 day-long GOLF time series that was also used in García et al. (2007) (see that paper for more details on the data analysis process). Figure 5 shows the results of the correlation between the reconstructed waves obtained from the GOLF data and those from the models. In this figure, instead of having one free parameter (physical processes or rotation), the results depend on both structure and dynamics. However, by mixing them, it is possible to extract some information on the data.

As in the case of the correlation with the reference model, we see a clear separation between the same two groups of models: On one side we have all the models using GN93 opacities and, on the other, the models using As05 opacities and the model without diffusion. This was an expected result. Indeed, we knew that GOLF is well correlated with the seismic model for rotation rates in the core not faster than five times - on average - the rotation rate in the rest of the radiative region whatever the considered fractional radius $\left(r_{i}\right)$. As we have seen in the previous section, the models containing the new abundances and the one 


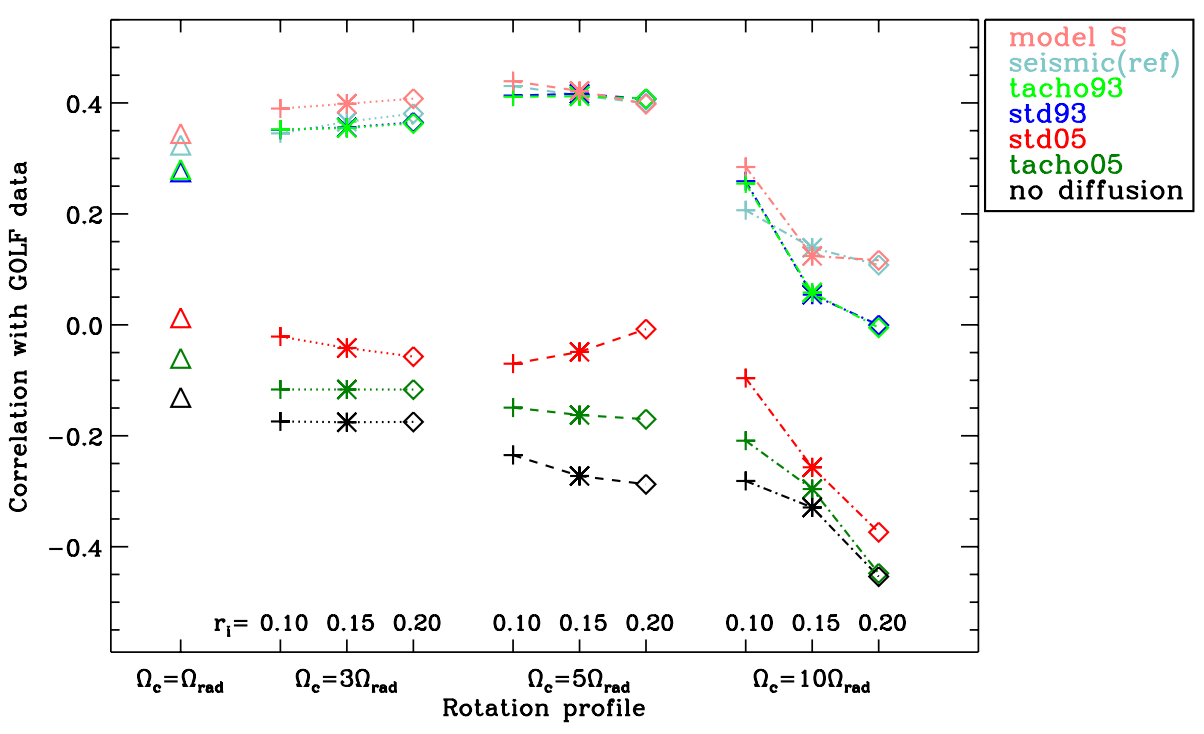

Figure 5 Correlation coefficients between the reconstructed waves from GOLF data and the ones from all of the models with the various rotation profiles. These models (described in Section 2) are std93 (blue), std05 (red), tacho93 (light green), tacho05 (dark green), no diffusion (black), model S (pink), and seismic (light blue).

without microscopic diffusion are incompatible with the models including the old chemical abundances. Therefore, they could not be compatible with the GOLF data. Actually, models that are close to the seismic model (that is, with the GN93 composition) associated with a rotation rate in the core $\Omega_{\mathrm{c}} \leq 5 \Omega_{\text {rad }}$ present the best compatibility with the data. A rotation profile having a rate five times the rotation rate in the rest of the radiative zone gives the highest correlation coefficients.

It is also important to note that the models using As05 and the one without treatment of the microscopic diffusion have, in most cases, negative correlations. The $g$-mode frequencies of these models are shifted as compared to GOLF and the maxima of the reconstructed waves are placed in between those of the GOLF data.

We know that the largest difference of $g$-mode frequencies can reach up to $5 \mu \mathrm{Hz}$ between the models with the GN93 abundances and the other group of models. In contrast to that, among the group of models presenting the highest correlations in Figure 5, the frequencies are shifted by less than $1 \mu \mathrm{Hz}$ (see Mathur et al., 2007). Therefore, we can say that using this technique on GOLF data, we are able to distinguish among structural parameters of the solar models (such as the abundances) when the frequency differences induced by these modifications are of the order of $1 \mu \mathrm{Hz}$ or even larger. If solar models that differ by various physical processes produce $g$-mode frequencies with differences lower than a microhertz, the present methodology would be unable to distinguish among them. To improve our detection capability we should enlarge the analyzed region toward lower frequencies, where we have seen that the $g$-mode periods are sensitive to the models. 


\section{Conclusion}

In the present paper, we have studied the changes in the signal reconstructed from the period separation $\Delta P_{1}$, which vary when the physics of the solar models are modified, and we have verified whether it was possible to detect these changes in the $g$-mode asymptotic signature with the present uncertainties.

By comparing our different models with the reference one, we have learned that the methodology presented here offers enough sensitivity to distinguish between two sets of models: diffusive models using the old GN93 abundances and models using the new As05 abundances and the one not including microscopic diffusion.

This work shows that the $g$-mode signature detected in GOLF data clearly favors the models including the old abundances from Grevesse and Noels (1993). In this sense, the analysis of the $g$-mode signature recently detected is in agreement with previous results coming from the $p$-mode analysis showing a larger discrepancy in the sound-speed profile when we use the new abundances from Asplund, Grevesse, and Sauval (2005).

However, we have to keep in mind that these results do not mean that the new abundances are incorrect. They only tell us that the classic models including them are not compatible with the observations. It could be possible to obtain a better agreement by changing other physical quantities of the models such as the opacities or the reaction rates or by including new processes to compensate for the effects of the reduction in the metallicity.

Acknowledgements The authors want to thank J. Christensen-Dalsgaard who provided us with the model $\mathrm{S}$ and the adipack code, J. Provost for having given us her frequencies with which we could compare the ones of our standard model and for having produced the seismic-model frequencies from her own oscillation code, and A. Eff-Darwich and S. Turck-Chièze for useful discussions and comments. The GOLF experiment is based upon a consortium of institutes (IAS, CEA/Saclay, Nice, and Bordeaux Observatories from France and IAC from Spain) involving numerous scientists and engineers, as enumerated in Gabriel et al. (1995). SOHO is a mission of international cooperation between ESA and NASA. This work has been partially funded by Grant No. AYA2004-04462 of the Spanish Ministry of Education and Culture and by the European Helio- and Asteroseismology Network (HELAS: http://www.helas-eu.org/), a major international collaboration funded by the European Commission's Sixth Framework Programme.

\section{References}

Andersen, B.N.: 1996, Theoretical amplitudes of solar g-modes. Astron. Astrophys. 312, 610-614.

Appourchaux, T., Fröhlich, C., Andersen, B., Berthomieu, G., Chaplin, W.J., Elsworth, Y., Finsterle, W., Gough, D.O., Hoeksema, J.T., Isaak, G.R., Kosovichev, A.G., Provost, J., Scherrer, P.H., Sekii, T., Toutain, T.: 2000, Observational upper limits to low-degree solar g-modes. Astrophys. J. 538, $401-414$. doi:10.1086/309124.

Asplund, M., Grevesse, N., Sauval, A.J.: 2005, The solar chemical composition. In: Barnes, T.G. III, Bash, F.N. (eds.) Cosmic Abundances as Records of Stellar Evolution and Nucleosynthesis CS-336, Astron. Soc. Pac., San Francisko, 25 - 38.

Bahcall, J.N., Serenelli, A.M., Basu, S.: 2005, New solar opacities, abundances, helioseismology, and neutrino fluxes. Astrophys. J. 621, L85 - L88. doi:10.1086/428929.

Basu, S., Christensen-Dalsgaard, J., Chaplin, W.J., Elsworth, Y., Isaak, G.R., New, R., Schou, J., Thompson, M.J., Tomczyk, S.: 1997, Solar internal sound speed as inferred from combined BiSON and LOWL oscillation frequencies. Mon. Not. Roy. Astron. Soc. 292, 243.

Basu, S., Chaplin, W.J., Elsworth, Y., New, R., Serenelli, A.M., Verner, G.A.: 2007, Solar abundances and helioseismology: Fine-structure spacings and separation ratios of low-degree p-modes. Astrophys. J. 655, 660-671. doi:10.1086/509820.

Brun, A.S., Turck-Chièze, S., Zahn, J.P.: 1999, Standard solar models in the light of new helioseismic constraints. II. Mixing below the convective zone. Astrophys. J. 525, 1032-1041. doi:10.1086/307932.

Chaplin, W.J., Elsworth, Y., Isaak, G.R., Marchenkov, K.I., Miller, B.A., New, R.: 2001, Rigid rotation of the solar core? On the reliable extraction of low-1 rotational p-mode splittings from full-disc observations of the Sun. Mon. Not. Roy. Astron. Soc. 327, 1127 -1136. doi:10.1046/j.1365-8711.2001.04805.x. 
Chaplin, W.J., Serenelli, A.M., Basu, S., Elsworth, Y., New, R., Verner, G.A.: 2007, Solar heavy-element abundance: constraints from frequency separation ratios of low-degree p-modes. Astrophys. J. 670, $872-$ 884. doi: $10.1086 / 522578$.

Christensen-Dalsgaard, J., Gough, D.O., Thompson, M.J.: 1991, The depth of the solar convection zone. Astrophys. J. 378, 413 - 437. doi:10.1086/170441.

Christensen-Dalsgaard, J., Dappen, W., Ajukov, S.V., Anderson, E.R., Antia, H.M., Basu, S., Baturin, V.A., Berthomieu, G., Chaboyer, B., Chitre, S.M., Cox, A.N., Demarque, P., Donatowicz, J., Dziembowski, W.A., Gabriel, M., Gough, D.O., Guenther, D.B., Guzik, J.A., Harvey, J.W., Hill, F., Houdek, G., Iglesias, C.A., Kosovichev, A.G., Leibacher, J.W., Morel, P., Proffitt, C.R., Provost, J., Reiter, J., Rhodes, E.J. Jr., Rogers, F.J., Roxburgh, I.W., Thompson, M.J., Ulrich, R.K.: 1996, The current state of solar modeling. Science 272, 1286-1292.

Couvidat, S., Turck-Chièze, S., Kosovichev, A.G.: 2003, Solar seismic models and the neutrino predictions. Astrophys. J. 599, 1434 - 1448. doi:10.1086/379604.

Couvidat, S., García, R.A., Turck-Chièze, S., Corbard, T., Henney, C.J., Jiménez-Reyes, S.: 2003, The rotation of the deep solar layers. Astrophys. J. 597, L77-L79. doi:10.1086/379698.

Domingo, V., Fleck, B., Poland, A.I.: 1995, The SOHO mission: an overview. Solar Phys. 162, 1-37.

Elsworth, Y.P., Baudin, F., Chaplin, W., Andersen, B., Appourchaux, T., Boumier, P., Broomhall, A.M., Corbard, T., Finsterle, W., Fröhlich, C., Gabriel, A., García, R.A., Gough, D.O., Grec, G., Jiménez, A., Kosovichev, A., Provost, J., Sekii, T., Toutain, T., Turck-Chièze, S.: 2006, The internal structure of the Sun inferred from g modes and low-frequency p modes. In: Fletcher, K., Thompson, M. (eds.) Proceedings of SOHO 18/GONG 2006/HELAS I, Beyond the spherical Sun SP-624, ESA, Noordwijk, 18.

Gabriel, A.H., Grec, G., Charra, J., Robillot, J.M., Cortés, T.R., Turck-Chièze, S., Bocchia, R., Boumier, P., Cantin, M., Céspedes, E., Cougrand, B., Cretolle, J., Dame, L., Decaudin, M., Delache, P., Denis, N., Duc, R., Dzitko, H., Fossat, E., Fourmond, J.J., García, R.A., Gough, D., Grivel, C., Herreros, J.M., Lagardere, H., Moalic, J.P., Pallé, P.L., Petrou, N., Sanchez, M., Ulrich, R., van der Raay, H.B.: 1995, Global oscillations at low frequency from the SOHO mission (GOLF). Solar Phys. 162, 61 - 99.

Gabriel, A.H., Baudin, F., Boumier, P., García, R.A., Turck-Chièze, S., Appourchaux, T., Bertello, L., Berthomieu, G., Charra, J., Gough, D.O., Pallé, P.L., Provost, J., Renaud, C., Robillot, J.M., Roca Cortés, T., Thiery, S., Ulrich, R.K.: 2002, A search for solar g modes in the GOLF data. Astron. Astrophys. 390, 1119 - 1131. doi:10.1051/0004-6361:20020695.

García, R.A., Turck-Chièze, S., Boumier, P., Robillot, J.M., Bertello, L., Charra, J., Dzitko, H., Gabriel, A.H., Jiménez-Reyes, S.J., Pallé, P.L., Renaud, C., Roca Cortés, T., Ulrich, R.K.: 2005, Global solar Doppler velocity determination with the GOLF/SoHO instrument. Astron. Astrophys. 442, $385-395$. doi:10.1051/0004-6361:20052779.

García, R.A., Turck-Chièze, S., Jiménez-Reyes, S.J., Ballot, J., Pallé, P.L., Eff-Darwich, A., Mathur, S., Provost, J.: 2007, Tracking solar gravity modes: the dynamics of the solar core. Science 316, 1591 1593. doi:10.1126/science.1140598.

Grevesse, N., Noels, A.: 1993, Cosmic abundances of the elements. In: Prantzos, N., Vangioni-Flam, E., Casse, M. (eds.) Origin and Evolution of the Elements 199, Cambridge, England, 15 - 25.

Guzik, J.A., Watson, L.S., Cox, A.N.: 2005, Can enhanced diffusion improve helioseismic agreement for solar models with revised abundances? Astrophys. J. 627, 1049-1056. doi:10.1086/430438.

Howe, R., Christensen-Dalsgaard, J., Hill, F., Komm, R.W., Larsen, R.M., Schou, J., Thompson, M.J., Toomre, J.: 2000, Dynamic variations at the base of the solar convection zone. Science 287, 24562460.

Kumar, P., Quataert, E.J., Bahcall, J.N.: 1996, Observational searches for solar g-modes: some theoretical considerations. Astrophys. J. 458, L83 - L85. doi:10.1086/309926.

Mathur, S.: 2007, A la recherche des modes de gravité: étude de la dynamique du coeur solaire. Ph.D. thesis, Université Paris XI Orsay.

Mathur, S., Turck-Chièze, S., Couvidat, S., García, R.A.: 2007, On the characteristics of the solar gravity mode frequencies. Astrophys. J. 668, 594-602. doi:10.1086/521187.

Michaud, G., Proffitt, C.R.: 1993, Particle transport processes. In: Weiss, W.W., Baglin, A. (eds.) IAU Colloq. 137: Inside the Stars CS-40, Astron. Soc. Pac., San Francisco, 246-259.

Morel, P.: 1997, CESAM: A code for stellar evolution calculations. Astron. Astrophys. Suppl. 124, $597-614$.

Morel, P., Provost, J., Berthomieu, G.: 1997, Updated solar models. Astron. Astrophys. 327, 349 - 360.

Press, W.H., Teukolsky, S.A., Vetterling, W.T., Flannery, B.P.: 1992, Numerical Recipes in FORTRAN. The Art of Scientific Computing, 2nd edn. Cambridge University Press, Cambridge.

Provost, J., Berthomieu, G.: 1986, Asymptotic properties of low degree solar gravity modes. Astron. Astrophys. 165, 218-226.

Spiegel, E.A., Zahn, J.P.: 1992, The solar tachocline. Astron. Astrophys. 265, $106-114$.

Tassoul, M.: 1980, Asymptotic approximations for stellar nonradial pulsations. Astrophys. J. Suppl. Ser. 43, 469 - 490. doi:10.1086/190678. 
Thompson, M.J., Toomre, J., Anderson, E., Antia, H.M., Berthomieu, G., Burtonclay, D., Chitre, S.M., Christensen-Dalsgaard, J., Corbard, T., Derosa, M., Genovese, C.R., Gough, D.O., Haber, D.A., Harvey, J.W., Hill, F., Howe, R., Korzennik, S.G., Kosovichev, A.G., Leibacher, J.W., Pijpers, F.P., Provost, J., Rhodes, E.J., Schou, J., Sekii, T., Stark, P.B., Wilson, P.: 1996, Differential rotation and dynamics of the solar interior. Science 272, 1300 - 1305.

Thompson, M.J., Christensen-Dalsgaard, J., Miesch, M.S., Toomre, J.: 2003, The internal rotation of the Sun. Annu. Rev. Astron. Astrophys. 41, 599-643. doi:10.1146/annurev.astro.41.011802.094848.

Turck-Chièze, S., Couvidat, S., Kosovichev, A.G., Gabriel, A.H., Berthomieu, G., Brun, A.S., ChristensenDalsgaard, J., García, R.A., Gough, D.O., Provost, J., Roca-Cortés, T., Roxburgh, I.W., Ulrich, R.K.: 2001, Solar neutrino emission deduced from a seismic model. Astrophys. J. 555, L69-L73. doi:10.1086/321726.

Turck-Chièze, S., Couvidat, S., Piau, L., Ferguson, J., Lambert, P., Ballot, J., García, R.A., Nghiem, P.: 2004a, Surprising Sun: a new step towards a complete picture? Phys. Rev. Lett. 93(21), 211102-1-211102-4. doi:10.1103/PhysRevLett.93.211102.

Turck-Chièze, S., García, R.A., Couvidat, S., Ulrich, R.K., Bertello, L., Varadi, F., Kosovichev, A.G., Gabriel, A.H., Berthomieu, G., Brun, A.S., Lopes, I., Pallé, P., Provost, J., Robillot, J.M., Roca Cortés, T.: 2004b, Looking for gravity-mode multiplets with the GOLF experiment aboard SOHO. Astrophys. J. 604, 455468. doi:10.1086/381743.

Zaatri, A., Provost, J., Berthomieu, G., Morel, P., Corbard, T.: 2007, Sensitivity of low degree oscillations to the change in solar abundances. Astron. Astrophys. 469, 1145-1149. doi:10.1051/ 0004-6361:20077212. 